\title{
Impact of a more restrictive overlapping surgery policy: an analysis of pre- and postimplementation complication rates, resident involvement, and surgical wait times at a high-volume neurosurgical department
}

\author{
Jian Guan, MD, Michael Karsy, MD, PhD, Andrea A. Brock, MD, MSCl, \\ William T. Couldwell, MD, PhD, John R. W. Kestle, MD, Randy L. Jensen, MD, PhD, \\ Andrew T. Dailey, MD, and Richard H. Schmidt, MD, PhD \\ Department of Neurosurgery, Clinical Neurosciences Center, University of Utah, Salt Lake City, Utah
}

OBJECTIVE Recently, overlapping surgery has been a source of controversy both in the popular press and within the academic medical community. There have been no studies examining the possible effects of more stringent overlapping surgery restrictions. At the authors' institution, a new policy was implemented that restricts attending surgeons from starting a second case until all critical portions of the first case that could require the attending surgeon's involvement are completed. The authors examined the impact of this policy on complication rates, neurosurgical resident education, and wait times for neurosurgical procedures.

METHODS The authors performed a retrospective chart review of nonemergency neurosurgical procedures performed over two periods-from June 1, 2014, to October 31, 2014 (pre-policy change) and from June 1, 2016, to October 31, 2016 (post-policy change) -by any of 4 senior neurosurgeons at a single institution who were authorized to schedule overlapping cases. Information on preoperative evaluation, patient demographics, premorbid conditions, surgical variables, and postoperative course were collected and analyzed.

RESULTS Six hundred fifty-three patients met inclusion criteria for complications analysis. Of these, $378(57.9 \%)$ underwent surgery before the policy change. On multivariable regression analysis, neither overlapping surgery (odds ratio $[\mathrm{OR}] 1.072,95 \%$ confidence interval $[\mathrm{Cl}]$ 0.710-1.620) nor the overlapping surgery policy change $(\mathrm{OR} 1.057,95 \% \mathrm{Cl}$ $0.700-1.596)$ was associated with overall complication rates. Similarly, neither overlapping surgery (OR $1.472,95 \% \mathrm{Cl}$ $0.883-2.454)$ nor the overlapping surgery policy change (OR 1.251, 95\% $\mathrm{Cl}$ 0.748-2.091) was associated with numbers of serious complications. After the policy change, the percentage of procedures in which the senior assistant was a postresidency fellow increased significantly, from $11.9 \%$ to $34.2 \%(p<0.001)$. In a multiple linear regression analysis of surgery wait times, patients undergoing surgery after the policy change had significantly longer delays from the decision to operate until the actual neurosurgical procedure $(p<0.001)$.

CONCLUSIONS At the authors' institution, further restriction of overlapping surgery was not associated with a reduction in overall or serious complications. Resident involvement in neurosurgical procedures decreased significantly after the policy change, and this study suggests that wait times for neurosurgical procedures also significantly lengthened. https://thejns.org/doi/abs/10.3171/2017.5.JNS17183

KEY WORDS overlapping surgery; complications; neurosurgery; outcome; wait times; resident training; surgical technique

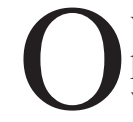
VERLAPPING surgery, defined as a single surgeon participating simultaneously in two operations in which the critical or key portions of each surgery occur at different times, ${ }^{3,4}$ has long been a common occurrence at academic medical centers nationwide. ${ }^{5}$ Following recent popular media reports on the practice, ${ }^{2}$ overlapping surgery has come under increased scrutiny. Despite the ubiquity of the practice, published data on the safety of overlapping surgery is scant, although an increasing body of evidence suggests that overlapping cases have a comparable safety profile with nonoverlapping procedures. ${ }^{7,9,14,15}$

Public response to the controversy has heralded a swift 
call to action by regulators. ${ }^{1}$ This has resulted in a US Senate Finance Committee investigation into overlapping surgery and the determination that further regulations are needed.$^{13}$ Many hospitals, including our own, have proactively adopted more restrictive policies concerning overlapping surgery in response to the early media reaction. It is unknown, however, what impact these policy changes may have; the long-held reasoning behind overlapping surgery is that doing so allows for improved surgical training for residents, readier patient access to surgeons (surgeon availability), and more extensive lifetime experience with particularly complex pathological conditions for subspecialists. ${ }^{8,12}$ To assess these effects, we performed a retrospective analysis of neurosurgical cases performed at our hospital before and after a more restrictive overlapping surgery policy (Table 1) was implemented. The primary differences between the old and new policies were the addition of a rule that an overlapping case could not be started until after completion of the critical portion of the first case and the requirement that the attending physician could not return to the first case after beginning a second. Our analysis focused specifically on complication rates, resident involvement, and wait times experienced by patients prior to surgical intervention.

\section{Methods \\ Study Population}

With approval from the University of Utah IRB, we performed a retrospective chart review of all cases over two time periods-from June 1, 2014, to October 31, 2014 (pre-policy change), and from June 1, 2016, to October 31, 2016 (post-policy change) - performed by any of 4 neurosurgeons at our institution who were authorized to run

\section{TABLE 1. Old and new overlapping surgery policies}

Old Policy (Prior to June 1, 2016)

The attending physician of record must be present and actively involved in any critical portions of the procedure.

The attending physician must be an associate professor or above in academic rank.

The attending physician must be board certified in neurosurgery.

The attending physician must have at least 5 years of experience within the University of Utah Department of Neurosurgery regardless of prior practice and/or training.

The attending physician must be a subspecialist in the area of the overlapping cases.

Qualified assistants of the appropriate training level must be available in both rooms.

New Policy (Beginning June 1, 2016)

All aspects of the old policy continue to apply.

The attending physician is responsible for the operative care of the patient throughout the operation.

Except for unanticipated circumstances as described below, the attending physician shall be physically available to the operating suite or in the immediate vicinity for the entire surgical procedure. Where more than 1 attending physician is necessary to complete only a portion of a patient's overall operative care, those attending physicians who provide only a portion of the surgical care are not required to stay for the duration of the operative care once their portion has been satisfactorily completed.

The attending physician who performs the procedure has the responsibility to inform the patient that he or she is performing the operation and to inform the patient what the designees' roles will be in the procedure.

The attending physician may not be scheduled to work in 2 operating rooms except under the following circumstances:

1) Staggering. Staggering is defined as conducting surgical procedures in 2 operating rooms in which the attending physician moves from the first room to the second without rescrubbing back into the first case. The attending physician is present in the operating room with the patient at the beginning of the procedure for the safety timeout and is present for all critical or key portions of the procedure until the point at which the attending physician determines his/her expertise is no longer necessary. The attending physician does not participate in a second case until the key or critical portions of the first procedure are completed and then can enter the second operating room to conduct the preincision timeout for the second case. This allows designees to open or close cases when the attending physician is not present. Staggering cases is only scheduled when there is sufficient operating room capacity.

2) Surgical procedures with multiple surgical teams involved. Surgical procedures may have more than 1 surgical team involved; in these cases, the surgical teams may schedule in other rooms during the periods of time they are not needed in the primary case. One surgical team cannot leave until the other team has arrived, that is, there must always be an attending physician or their designee in the room at all times.

3) Surgical procedures with multiple surgeons for same team involved. Surgical procedures may have more than 1 surgeon from the same team involved; in these cases, all attending physicians' names must be listed on the consent form. One attending physician must be present at the beginning of the case and at minimum 1 surgeon will be scrubbed in the case until the key or critical portions of the procedure are completed.

A primary attending physician may have to leave the operating room for a procedure-related task. Such procedure-related tasks could include review of pertinent pathology ("frozen section") and diagnostic imaging; a discussion with the patient's family; and breaks during long procedures. The surgeon must be immediately available for recall during such absences.

The attending physician may designate part of the patient's care to a designee provided the attending physician is present for all critical or key portions of the operation.

The designee has the responsibility for informing the respective attending physician who has ultimate responsibility for those components of the patient's care, before undertaking operative care. The attending anesthesiologist must be present for the induction of all general anesthetics and the attending physician must be present for the preincision timeout as stated in University of Utah hospital policy. 
overlapping surgeries during both timeframes. These dates were chosen to capture the period of time immediately after the new overlapping policy went into effect (from June 1,2016 , onward) and the equivalent calendar period of the year prior to publication of the initial Boston Globe article surveying overlapping surgery, which was published in 2015. ${ }^{2}$ Cases involving patients younger than 10 years of age were excluded from this study, as were cases performed on weekends as they are never performed in an overlapping manner. "Emergency" cases were excluded because they were almost exclusively nonoverlapping and they have an inherently high rate of adverse events due to the patient condition on presentation. In the rare instance in which an emergency case overlapped with an elective or urgent case, the elective or urgent case was also excluded from analysis.

\section{Data Collection}

A comprehensive review of the medical record was conducted, including preoperative clinic notes, operative reports, inpatient progress notes, discharge summaries, and follow-up clinic notes. Patient characteristics such as demographics, comorbidities, operative variables, postoperative course, and complications were recorded for each case.

Demographic variables collected included age, sex, race and/or ethnicity, body mass index (BMI), and insurance type (private, Medicaid/self-pay, or Medicare). Comorbidities included each patient's American Society of Anesthesiologists (ASA) physical classification; active tobacco, alcohol, or illicit drug use; preoperative corticosteroid use; cardiovascular comorbidities (e.g., atrial fibrillation, history of myocardial infarction); pulmonary comorbidities (e.g., obstructive sleep apnea, asthma); hepatobiliary comorbidities (e.g., cirrhosis, hepatitis); renal comorbidities (e.g., chronic kidney disease); diabetes; and hypertension. Each patient was also assessed for neurological comorbidities (e.g., stroke, multiple sclerosis) and oncological conditions (i.e., the presence of active cancer or ongoing cancer treatment), but these were only included if the neurological or oncological condition was not the indication for neurosurgical intervention.

Operative variables consisted of surgery type (separated into 1 of 10 categories), attending neurosurgeon (dichotomized based on $>20$ or $<20$ years of postresidency practice), the level of training of the most senior resident or fellow assisting in the neurosurgical procedure, the start time of the operation (defined based on the start of anesthesia), the duration of surgery (defined based on the start and end times of anesthesia), and intraoperative blood loss. Cases were also categorized as elective or urgent cases; elective cases were defined as operations scheduled more than 24 hours prior to the start of surgery, whereas urgent cases began less than 24 hours after being added to the schedule but were not immediately life-threatening in nature. Postoperative course data included length of hospitalization postoperatively and discharge destination.

Each case was dichotomized into overlapping or nonoverlapping, with overlapping cases defined as any case in which 2 patients under the care of a single lead surgeon were under anesthesia at the same time. The amount of time during which cases overlapped was also collected.

Similar to our previous study, ${ }^{7}$ a complication was de- fined as any deviation from the expected intraoperative or postoperative course. Complications were separated into intraoperative, postoperative, and those occurring within 30 days of discharge. Complication severity was graded based on the scale proposed by Landriel Ibañez et al. ${ }^{10}$ In this scale, complications are separated into 4 categories: Grade I, complications that are not life-threatening and do not require invasive treatment; Grade II, events requiring invasive surgical, endoscopic, or endovascular intervention; Grade III, life-threatening events that require intensive care management; and Grade IV, complications resulting in death. For our analysis, Grade 0 was given to cases without complications. In patients with multiple complications during their hospitalization, the highestgrade complication was used for analysis.

Finally, the wait time for each neurosurgical procedure was calculated. This was defined, as previously described, ${ }^{6}$ as the number of days between the decision to operateidentified based on preoperative clinic notes - and the neurosurgical procedure. Cases in which the patient was admitted from the emergency department, was directly admitted to the hospital for surgery from clinic, or was referred from another inpatient service for surgery during the same hospitalization were excluded from wait time analysis because of the unplanned nature of these surgeries.

\section{Statistical Analysis}

Continuous variables in all cases were analyzed using the Student t-test, whereas categorical variables were analyzed using chi-square analysis. Univariate analysis was first performed comparing cases undertaken before the policy change with those performed after the policy change. We then completed univariate analysis to evaluate factors associated with any complications or serious (Grade II or higher) complications during the operative, postoperative, and 30-day postdischarge period.

To further assess the influence of the policy change on complication rates, we performed a pair of multivariable logistic regression analyses. Variables with a p value $<0.2$ on univariate analysis were included in forward, stepwise, multivariable regression models with an entry cutoff of 0.05 and a removal cutoff of 0.20 . A separate model was constructed for both overall complications and serious complications. The pre- or post-policy change variable was included in both multivariable models regardless of the $\mathrm{p}$ value, as was the overlapping surgery variable.

Finally, to evaluate the effect of the policy change on wait times for neurosurgical procedures, we performed a multiple linear regression. Variables included in the linear regression model were chosen by the investigators before the analysis based on their possible influence on surgical wait times. These consisted of the surgery category, individual attending physician, insurance type, and policychange status. In all analyses, $\mathrm{p}<0.05$ was considered statistically significant. All statistical analysis was performed using SPSS version 20.0 (IBM Corp.).

\section{Results \\ Patient Cohort}

Of 695 cases performed by the 4 neurosurgeons during 
the study period, 653 (94.0\%) met the criteria for inclusion. Twenty-three cases were excluded because they were classified as emergent, 16 cases occurred on a weekend, 2 cases were excluded because the patient was younger than 10 years of age at the time of surgery, and 1 elective case was excluded because it overlapped with an emergency case.

For our wait time analysis, 184 additional cases were excluded-143 admitted for surgery from the emergency department, 26 inpatient referrals, and 15 directly admitted from clinic. This left 469 total cases for wait time analysis-265 cases before the policy change and 204 cases after the policy change.

\section{Pre-Policy Versus Post-Policy Change Analysis}

Over the study period, 378 neurosurgical cases were performed by the 4 included attending surgeons before the policy change, whereas 275 neurosurgical cases were performed after the policy change (Table 2). Patients who underwent surgery before the policy change were significantly older than those operated on after the policy change $(53.8 \pm 16.2$ vs $49.2 \pm 17.5$ years old, $p=0.001)$. They were also more likely to be male ( $51.3 \%$ vs $38.5 \%, \mathrm{p}=0.001)$ and to use Medicare (38.4\% vs $29.8 \%, \mathrm{p}=0.036$ ). Prepolicy change patients were also less likely to have ASA Class I or II (44.4\% vs $56.7 \%, \mathrm{p}<0.001)$ and were more likely to have a neurological comorbidity $(17.2 \%$ vs $8.7 \%$, $\mathrm{p}=0.002)$ or oncological condition $(11.9 \%$ vs $4.4 \%, \mathrm{p}=$ $0.001)$. Although there was no significant difference in the types of cases performed during the pre- and post-policy change periods $(p=0.525)$, the rate of overlapping surgery, as expected, decreased significantly after the more restrictive overlapping surgery policy was implemented $(46.0 \%$ vs $26.9 \%$, p < 0.001 ), as did the average time during which cases overlapped $(193.3 \pm 104.2$ minutes vs $154.5 \pm 110.6$ minutes, $\mathrm{p}=0.009$ ). The proportion of cases classified as "urgent" also decreased after the policy change (11.6\% vs $5.8 \%, \mathrm{p}=0.011)$. There was no significant change in the overall complication rate with the policy change $(27.0 \%$ pre-policy change vs $27.6 \%$ post-policy change, $\mathrm{p}=$ $0.853)$, nor was there a change in the rate of serious complications $(13.2 \%$ pre-policy change vs $14.2 \%$ post-policy change, $p=0.726$ ). There was, however, a significant increase in the proportion of cases in which fellows were involved (11.9\% pre-policy change vs $34.2 \%$ post-policy change, $\mathrm{p}<0.001)$.

\section{All Complications Analysis}

Patients experiencing complications after surgery had significantly higher ASA classes than those who did not ( $\mathrm{p}$ $<0.001$ ) and were more likely to have a chief resident (postgraduate year $[\mathrm{PGY}] 7$ ) or fellow assisting the attending surgeon in their cases $(p=0.016)$. Craniotomies for tumors had significantly higher rates of overall complications than other procedures $(\mathrm{p}<0.001)$. Surgeries associated with complications lasted significantly longer than those that did not $(346.4 \pm 167.6$ minutes vs $258.6 \pm 114.5$ minutes, $\mathrm{p}<0.001)$ and resulted in significantly more blood loss $(368.8 \pm 591.8 \mathrm{ml}$ vs $138.5 \pm 181.8 \mathrm{ml}, \mathrm{p}<0.001)$. Patients who experienced complications had significantly longer hospital stays $(7.5 \pm 6.0$ days vs $3.2 \pm 3.3$ days, $\mathrm{p}<0.001)$ and were significantly less likely to be discharged home $(41.0 \%$ vs $80.0 \%, \mathrm{p}<0.001)$. There was no significant difference in rates of overlapping surgery between patients who experienced complications and those who did not ( $\mathrm{p}$ $=0.772$ ), nor was there a difference in whether they were operated on before or after the policy change $(p=0.853)$.

In the multivariable model for any complications (Table 3 ), surgery type remained significantly associated with the occurrence of any complication $(\mathrm{p}=0.041)$, as did surgery length (OR 1.003, 95\% CI 1.000-1.005), estimated blood loss during the case (OR 1.002, 95\% CI 1.001-1.003), preoperative hepatobiliary comorbidity (OR 2.628, 95\% CI 1.032-6.693), and higher ASA class ( $\mathrm{p}<0.001)$. Neither overlapping surgery (OR $1.072,95 \%$ CI $0.710-1.620)$ nor having surgery after the policy change (OR 1.057, 95\% CI 0.700-1.596) was associated with the occurrence of a complication.

To account for the range of overlap times between overlapping surgeries, we ran a supplemental analysis using the overlapping percentage (overlap time divided by total surgery duration) in our multivariable model of overall complications in place of the overlapping surgery variable. This had no influence on the factors significantly associated with the overall complication rate; specifically, the association of overlapping percentage $(\mathrm{p}=0.955)$ and overlapping surgery policy change with overall complications $(\mathrm{p}=0.860)$ remained nonsignificant.

\section{Serious Complications Analysis}

Patients experiencing serious complications after surgery had significantly higher ASA classes than those who did not ( $\mathrm{p}<0.001$; Table 1). They were also more likely to have used corticosteroids preoperatively $(21.3 \%$ vs $13.3 \%$, $\mathrm{p}=0.044$ ) but were less likely to have used illicit drugs $(1.1 \%$ vs $6.7 \%, p=0.038)$. Patients suffering serious complications were also more likely to have renal comorbidities $(5.6 \%$ vs $2.0 \%, \mathrm{p}=0.038)$ and neurological comorbidities $(21.3 \%$ vs $12.4 \%, \mathrm{p}=0.022)$. Craniotomies for tumors had significantly higher rates of serious complications than other surgical categories, whereas both instrumented and noninstrumented spinal procedures had significantly lower rates $(\mathrm{p}<0.001)$. Surgeries associated with serious complications lasted significantly longer $(344.1 \pm 177.0$ minutes vs $272.8 \pm 126.7$ minutes, $\mathrm{p}<0.001$ ), were associated with higher blood loss $(277.4 \pm 353.4 \mathrm{ml}$ vs $189.3 \pm$ $359.9 \mathrm{ml}, \mathrm{p}=0.032)$, and ended later in the day $(15: 49 \pm$ $3: 56$ vs $14: 52 \pm 3: 13, p=0.014)$. Patients experiencing serious complications stayed significantly longer in the hospital after surgery compared with those who did not $(9.0$ \pm 7.3 days vs $3.6 \pm 3.5$ days, $p<0.001)$ and were significantly less likely to be discharged home $(33.7 \%$ vs $75.0 \%$, $\mathrm{p}<0.001)$. There was no significant difference in rates of overlapping surgery between patients who experienced serious complications and those who did not $(\mathrm{p}=0.324)$, nor was there a difference in whether they were operated on before or after the policy change $(\mathrm{p}=0.726)$.

In the multivariable model for serious complications (Table 4), surgery type remained significantly associated with the occurrence of any complication $(\mathrm{p}=0.049)$, as did surgery length (OR 1.004, 95\% CI 1.002-1.006) and higher ASA class $(\mathrm{p}<0.001)$. Neither overlapping surgery 
TABLE 2. Univariate analyses of policy change, any complication, and serious complications

\begin{tabular}{|c|c|c|c|c|c|c|c|c|c|}
\hline \multirow[b]{2}{*}{ Variable } & \multirow{2}{*}{$\begin{array}{l}\text { Pre-Policy } \\
\text { Change }\end{array}$} & \multirow{2}{*}{$\begin{array}{l}\text { Post-Policy } \\
\text { Change }\end{array}$} & \multirow{2}{*}{$\begin{array}{c}p \\
\text { Value }\end{array}$} & \multicolumn{2}{|c|}{ Complication } & \multirow{2}{*}{$\begin{array}{c}p \\
\text { Value }\end{array}$} & \multicolumn{2}{|c|}{ Serious Complication* } & \multirow{2}{*}{$\begin{array}{c}\mathrm{p} \\
\text { Value }\end{array}$} \\
\hline & & & & None & Any & & No & Yes & \\
\hline No. of patients & 378 & 275 & & 475 & 178 & & 564 & 89 & \\
\hline Mean age in yrs $\pm S D$ & $53.8 \pm 16.2$ & $49.2 \pm 17.5$ & 0.001 & $51.2 \pm 17.1$ & $53.8 \pm 16.1$ & 0.078 & $51.5 \pm 17.1$ & $54.3 \pm 15.2$ & 0.146 \\
\hline Male sex & $194(51.3)$ & $106(38.5)$ & 0.001 & $221(46.5)$ & $79(44.4)$ & 0.624 & $263(46.6)$ & $37(41.6)$ & 0.347 \\
\hline \multicolumn{10}{|l|}{ Race/ethnicity } \\
\hline Caucasian & $339(89.7)$ & $238(86.5)$ & \multirow{7}{*}{0.751} & $422(88.8)$ & $155(87.1)$ & \multirow{7}{*}{0.176} & $493(87.4)$ & $84(94.4)$ & \multirow{7}{*}{0.530} \\
\hline Hispanic/Latino & $19(5.0)$ & $17(6.2)$ & & $26(5.5)$ & $10(5.6)$ & & $33(5.9)$ & $3(3.4)$ & \\
\hline African American & $1(0.3)$ & $2(0.7$ & & $3(0.6)$ & $0(0)$ & & $3(0.5)$ & $0(0)$ & \\
\hline Asian & $4(1.1)$ & $3(1.1)$ & & $5(1.1)$ & $2(1.1)$ & & $7(1.2)$ & $0(0)$ & \\
\hline American Indian & $3(0.8)$ & $2(0.7)$ & & $2(0.4)$ & $3(1.7)$ & & $4(0.7)$ & $1(1.1)$ & \\
\hline $\begin{array}{l}\text { Hawaiian/Pacific } \\
\text { Islander }\end{array}$ & $4(1.1)$ & $2(0.7)$ & & $2(0.4)$ & $4(2.2)$ & & $6(1.1)$ & $0(0)$ & \\
\hline Other/unknown & $8(2.1)$ & $11(4.0)$ & & $15(3.2)$ & $4(2.2)$ & & $18(3.2)$ & $1(1.1)$ & \\
\hline Mean BMI $\pm \mathrm{SD}\left(\mathrm{kg} / \mathrm{m}^{2}\right)$ & $28.6 \pm 6.7$ & $28.8 \pm 7.0$ & 0.601 & $28.7 \pm 6.5$ & $28.6 \pm 7.7$ & 0.848 & $28.7 \pm 6.7$ & $28.4 \pm 7.6$ & 0.696 \\
\hline \multicolumn{10}{|l|}{ Insurance type } \\
\hline Private & $203(53.7)$ & $160(58.2)$ & \multirow{3}{*}{0.036} & $272(57.3)$ & $91(51.1)$ & \multirow{3}{*}{0.228} & $314(55.7)$ & $49(55.1)$ & \multirow{3}{*}{0.387} \\
\hline Medicaid/self-pay & $30(7.9)$ & $33(12.0)$ & & $41(8.6)$ & $22(12.4)$ & & $51(9.0)$ & $12(13.5)$ & \\
\hline Medicare & $145(38.4)$ & $82(29.8)$ & & $162(34.1)$ & $65(36.5)$ & & $199(35.3)$ & $28(31.5)$ & \\
\hline \multicolumn{10}{|l|}{ ASA Class } \\
\hline | or II & $168(44.4)$ & $156(56.7)$ & \multirow{3}{*}{$<0.001$} & $262(55.2)$ & $62(34.8)$ & \multirow{3}{*}{$<0.001$} & $298(52.8)$ & $26(29.2)$ & \multirow{3}{*}{$<0.001$} \\
\hline III & $187(49.5)$ & $93(33.8)$ & & $195(41.1)$ & $85(47.8)$ & & $237(42.0)$ & $43(48.3)$ & \\
\hline IV or V & $23(6.1)$ & $26(9.5)$ & & $18(3.8)$ & $31(17.4)$ & & $29(5.1)$ & $20(22.5)$ & \\
\hline Tobacco use & $39(10.3)$ & $29(10.5)$ & 0.925 & $53(11.2)$ & $15(8.4)$ & 0.309 & $61(10.8)$ & $7(7.9)$ & 0.397 \\
\hline Alcohol use & $124(32.8)$ & $99(36.0)$ & 0.395 & $166(34.9)$ & $57(32.0)$ & 0.483 & $191(33.9)$ & $32(36.0)$ & 0.699 \\
\hline Illicit drug use & $22(5.8)$ & $17(6.2)$ & 0.847 & $32(6.7)$ & $7(3.9)$ & 0.178 & $38(6.7)$ & $1(1.1)$ & 0.038 \\
\hline Corticosteroid use & $55(14.6)$ & $39(14.2)$ & 0.895 & $61(12.8)$ & $33(18.5)$ & 0.065 & $75(13.3)$ & $19(21.3)$ & 0.044 \\
\hline Cardiovascular comorbidity & $59(15.6)$ & $37(13.5)$ & 0.443 & $70(14.7)$ & $26(14.6)$ & 0.967 & $86(15.2)$ & $10(11.2)$ & 0.321 \\
\hline Pulmonary comorbidity & $120(31.7)$ & $95(34.5)$ & 0.452 & $156(32.8)$ & 59 (33.1) & 0.941 & $188(33.3)$ & $27(30.3)$ & 0.576 \\
\hline Hepatobiliary comorbidity & $16(4.2)$ & $6(2.2)$ & 0.152 & $12(2.5)$ & $10(5.6)$ & 0.051 & $20(3.5)$ & $2(2.2)$ & 0.528 \\
\hline Renal comorbidity & $6(1.6)$ & $10(3.6)$ & 0.094 & $9(1.9)$ & $7(3.9)$ & 0.134 & $11(2.0)$ & $5(5.6)$ & 0.038 \\
\hline Neurological comorbidity & $65(17.2)$ & $24(8.7)$ & 0.002 & $59(12.4)$ & $30(16.9)$ & 0.142 & $70(12.4)$ & $19(21.3)$ & 0.022 \\
\hline Oncological condition & $45(11.9)$ & $12(4.4)$ & 0.001 & $39(8.2)$ & $18(10.1)$ & 0.443 & $49(8.7)$ & $8(9.0)$ & 0.926 \\
\hline Diabetes & $38(10.1)$ & $33(12.0)$ & 0.430 & $49(10.3)$ & $22(12.4)$ & 0.455 & $59(10.5)$ & $12(13.5)$ & 0.395 \\
\hline Hypertension & $139(36.8)$ & $85(30.9)$ & 0.119 & $156(32.8)$ & $68(38.2)$ & 0.199 & $191(33.9)$ & $33(37.1)$ & 0.553 \\
\hline \multicolumn{10}{|l|}{ Surgery category } \\
\hline $\begin{array}{l}\text { Simple craniotomy for } \\
\text { tumor }\end{array}$ & $59(15.6)$ & $39(14.2)$ & \multirow{10}{*}{0.525} & $62(13.1)$ & $36(20.2)$ & & $75(13.3)$ & $23(25.8)$ & \\
\hline $\begin{array}{l}\text { Complex craniotomy for } \\
\text { tumor }\end{array}$ & $17(4.5)$ & $19(6.9)$ & & $16(3.4)$ & $20(11.2)$ & & $25(4.4)$ & $11(12.4)$ & \\
\hline $\begin{array}{l}\text { Craniotomy for } \\
\text { aneurysm/vascular } \\
\text { malformation }\end{array}$ & $19(5.0)$ & $8(2.9)$ & & $15(3.2)$ & $12(6.7)$ & & $20(3.5)$ & $7(7.9)$ & \\
\hline Craniotomy for other & $18(4.8)$ & $19(6.9)$ & & $30(6.3)$ & $7(3.9)$ & $<0.001$ & $32(5.7)$ & $5(5.6)$ & $<0.001$ \\
\hline Burr hole craniotomy & $13(3.4)$ & $6(2.2)$ & & $12(2.5)$ & $7(3.9)$ & & $13(2.3)$ & $6(6.7)$ & \\
\hline Noninstrumented spine & $44(11.6)$ & $38(13.8)$ & & $76(16.0)$ & $6(3.4)$ & & $79(14.0)$ & $3(3.4)$ & \\
\hline Instrumented spine & $76(20.1)$ & $62(22.5)$ & & $96(20.2)$ & $42(23.6)$ & & $128(22.7)$ & $10(11.2)$ & \\
\hline $\begin{array}{l}\text { Transnasal approach for } \\
\text { tumor }\end{array}$ & $31(8.2)$ & $21(7.6)$ & & $35(7.4)$ & $17(9.6)$ & & $47(8.3)$ & $5(5.6)$ & \\
\hline Shunt & $47(12.4)$ & $32(11.6)$ & & $67(14.1)$ & $12(6.7)$ & & $71(12.6)$ & $8(9.0)$ & \\
\hline Miscellaneous & $54(14.3)$ & $31(11.3)$ & & $66(13.9)$ & $19(10.7)$ & & $74(13.1)$ & $11(12.4)$ & \\
\hline
\end{tabular}


» CONTINUED FROM PAGE 519

TABLE 2. Univariate analyses of policy change, any complication, and serious complications

\begin{tabular}{|c|c|c|c|c|c|c|c|c|c|}
\hline \multirow[b]{2}{*}{ Variable } & \multirow{2}{*}{$\begin{array}{l}\text { Pre-Policy } \\
\text { Change }\end{array}$} & \multirow{2}{*}{$\begin{array}{l}\text { Post-Policy } \\
\text { Change }\end{array}$} & \multirow{2}{*}{$\begin{array}{c}\mathrm{p} \\
\text { Value }\end{array}$} & \multicolumn{2}{|c|}{ Complication } & \multirow{2}{*}{$\begin{array}{c}\mathrm{p} \\
\text { Value }\end{array}$} & \multicolumn{2}{|c|}{ Serious Complication* } & \multirow{2}{*}{$\begin{array}{c}p \\
\text { Value }\end{array}$} \\
\hline & & & & None & Any & & No & Yes & \\
\hline Senior attending $\dagger$ & $188(49.7)$ & $140(50.9)$ & 0.767 & $239(50.3)$ & $89(50.0)$ & 0.943 & $279(49.5)$ & $49(55.1)$ & 0.327 \\
\hline Overlapping surgery & $174(46.0)$ & $74(26.9)$ & $<0.001$ & $182(38.3)$ & $66(37.1)$ & 0.772 & $210(37.2)$ & $38(42.7)$ & 0.324 \\
\hline Post-policy change & $0(0)$ & $275(100)$ & NA & $199(41.9)$ & $76(42.7)$ & 0.853 & $236(41.8)$ & $39(43.8)$ & 0.726 \\
\hline \multicolumn{10}{|l|}{ Scheduling type } \\
\hline Elective & $334(88.4)$ & $259(94.2)$ & \multirow{2}{*}{0.011} & $434(91.4)$ & $159(89.3)$ & \multirow{2}{*}{0.421} & $514(91.1)$ & $79(88.8)$ & \multirow{2}{*}{0.472} \\
\hline Urgent & $44(11.6)$ & $16(5.8)$ & & $41(8.6)$ & $19(10.7)$ & & $50(8.9)$ & $10(11.2)$ & \\
\hline \multicolumn{10}{|l|}{ Assistant training level } \\
\hline PGY-1 & $5(1.3)$ & $6(2.2)$ & \multirow{8}{*}{$<0.001$} & $8(1.7)$ & $3(1.7)$ & \multirow{8}{*}{0.016} & $9(1.6)$ & $2(2.2)$ & \multirow{8}{*}{0.729} \\
\hline PGY-2 & $30(7.9)$ & $6(2.2)$ & & $30(6.3)$ & $6(3.4)$ & & $31(5.5)$ & $5(5.6)$ & \\
\hline PGY-3 & $4(1.1)$ & $15(5.5)$ & & $15(3.2)$ & $4(2.2)$ & & $16(2.8)$ & $3(3.4)$ & \\
\hline PGY-4 & $6(1.6)$ & $11(4.0)$ & & $14(2.9)$ & $3(1.7)$ & & $15(2.7)$ & $2(2.2)$ & \\
\hline PGY-5 & $92(24.3)$ & $34(12.4)$ & & $103(21.7)$ & $23(12.9)$ & & $111(19.7)$ & $15(16.9)$ & \\
\hline PGY-6 & $75(19.8)$ & $24(8.7)$ & & $75(15.8)$ & $24(13.5)$ & & $91(16.1)$ & $8(9.0)$ & \\
\hline PGY-7 & $121(32.0)$ & $85(30.9)$ & & $143(30.1)$ & $63(35.4)$ & & $174(30.9)$ & $32(36.0)$ & \\
\hline Fellow & $45(11.9)$ & $94(34.2)$ & & 87 (18.3) & $52(29.2)$ & & $117(20.7)$ & $22(24.7)$ & \\
\hline Start time \pm SD & $10: 31 \pm 3: 21$ & $10: 04 \pm 3: 08$ & 0.081 & $10: 23 \pm 3: 14$ & $10: 10 \pm 3: 20$ & 0.436 & $10: 20 \pm 3: 12$ & $10: 21 \pm 3: 40$ & 0.965 \\
\hline End time \pm SD & $15: 05 \pm 3: 24$ & $14: 53 \pm 3: 15$ & 0.455 & $14: 42 \pm 3: 10$ & $15: 48 \pm 3: 38$ & $<0.001$ & $14: 52 \pm 3: 13$ & $15: 49 \pm 3: 56$ & 0.014 \\
\hline Surgery length (mins) $\pm S D$ & $277.7 \pm 140.9$ & $289.2 \pm 130.6$ & 0.290 & $258.6 \pm 114.5$ & $346.4 \pm 167.6$ & $<0.001$ & $272.8 \pm 126.7$ & $344.1 \pm 177.0$ & $<0.001$ \\
\hline Overlap time (mins) \pm SD & $193.3 \pm 104.2$ & $154.5 \pm 110.6$ & 0.009 & $175.4 \pm 102.0$ & $199.1 \pm 120.1$ & 0.124 & $179.1 \pm 105.9$ & $195.9 \pm 116.1$ & 0.376 \\
\hline $\begin{array}{l}\text { Estimated blood loss (ml) } \\
\quad \pm \text { SD }\end{array}$ & $205.5 \pm 411.1$ & $195.4 \pm 275.4$ & 0.723 & $138.5 \pm 181.8$ & $368.8 \pm 591.8$ & $<0.001$ & $189.3 \pm 359.9$ & $277.4 \pm 353.4$ & 0.032 \\
\hline Length of stay (days) \pm SD & $4.5 \pm 5.0$ & $4.2 \pm 3.9$ & 0.361 & $3.2 \pm 3.3$ & $7.5 \pm 6.0$ & $<0.001$ & $3.6 \pm 3.5$ & $9.0 \pm 7.3$ & $<0.001$ \\
\hline \multicolumn{10}{|l|}{ Discharge destination } \\
\hline Home & $252(66.7)$ & $201(73.1)$ & \multirow{7}{*}{0.086} & $380(80.0)$ & $73(41.0)$ & \multirow{7}{*}{$<0.001$} & $423(75.0)$ & $30(33.7)$ & \multirow{7}{*}{$<0.001$} \\
\hline Home health & $18(4.8)$ & $13(4.7)$ & & $24(5.1)$ & $7(3.9)$ & & $30(5.3)$ & $1(1.1)$ & \\
\hline Acute rehab & $60(15.9)$ & $43(15.6)$ & & $38(8.0)$ & $65(36.5)$ & & $65(11.5)$ & $38(42.7)$ & \\
\hline SNF & $31(8.2)$ & $8(2.9)$ & & $24(5.1)$ & $15(8.4)$ & & $32(5.7)$ & $7(7.9)$ & \\
\hline LTAC & $12(3.2)$ & $6(2.2)$ & & $7(1.5)$ & $11(6.2)$ & & $11(2.0)$ & $7(7.9)$ & \\
\hline Death & $3(0.8)$ & $4(1.5)$ & & $1(0.2)$ & $6(3.4)$ & & $1(0.2)$ & $6(6.7)$ & \\
\hline Other & $2(0.5)$ & $0(0)$ & & $1(0.2)$ & $1(0.6)$ & & $2(0.4)$ & $0(0)$ & \\
\hline Intraop complications & $21(5.6)$ & $14(5.1)$ & 0.795 & $0(0)$ & $35(19.7)$ & - & $25(4.4)$ & $10(11.2)$ & - \\
\hline \multicolumn{10}{|l|}{ Intraop complication grade } \\
\hline 0 & $357(94.4)$ & $261(94.9)$ & & $475(100.0)$ & $143(80.3)$ & & $539(95.6)$ & $79(88.8)$ & \\
\hline I & $17(4.5)$ & $13(4.7)$ & & $0(0)$ & $30(16.9)$ & & $25(4.4)$ & $5(5.6)$ & \\
\hline II & $1(0.3)$ & $0(0)$ & 0.814 & $0(0)$ & $1(0.6)$ & - & $0(0)$ & $1(1.1)$ & - \\
\hline III & $2(0.5)$ & $1(0.4)$ & & $0(0)$ & $3(1.7)$ & & $0(0)$ & $3(3.4)$ & \\
\hline IV & $1(0.3)$ & $0(0)$ & & $0(0)$ & $1(0.6)$ & & $0(0)$ & $1(1.1)$ & \\
\hline Postop complications & $70(18.5)$ & $53(19.3)$ & 0.808 & $0(0)$ & $123(69.1)$ & - & 64 (11.3) & $59(66.3)$ & - \\
\hline Postop complication grade & & & & & & & & & \\
\hline 0 & $308(81.5)$ & $222(80.7)$ & & $475(100.0)$ & $55(30.9)$ & & $500(88.7)$ & $30(33.7)$ & \\
\hline I & $37(9.8)$ & $30(10.9)$ & & $0(0)$ & $67(37.6)$ & & $64(11.3)$ & $3(3.4)$ & \\
\hline$\|$ & $6(1.6)$ & $7(2.5)$ & 0.798 & $0(0)$ & $13(7.3)$ & - & $0(0)$ & $13(14.6)$ & - \\
\hline III & $25(6.6)$ & $14(5.1)$ & & $0(0)$ & $39(21.9)$ & & $0(0)$ & $39(43.8)$ & \\
\hline IV & $2(0.5)$ & $2(0.7)$ & & $0(0)$ & $4(2.2)$ & & $0(0)$ & $4(4.5)$ & \\
\hline 30-day complications & $31(8.2)$ & $19(6.9)$ & 0.540 & $0(0)$ & $50(28.1)$ & - & $15(2.7)$ & $35(39.3)$ & - \\
\hline
\end{tabular}


» CONTINUED FROM PAGE 520

TABLE 2. Univariate analyses of policy change, any complication, and serious complications

\begin{tabular}{|c|c|c|c|c|c|c|c|c|c|}
\hline \multirow[b]{2}{*}{ Variable } & \multirow{2}{*}{$\begin{array}{c}\text { Pre-Policy } \\
\text { Change }\end{array}$} & \multirow{2}{*}{$\begin{array}{c}\text { Post-Policy } \\
\text { Change }\end{array}$} & \multirow{2}{*}{$\begin{array}{c}p \\
\text { Value }\end{array}$} & \multicolumn{2}{|c|}{ Complication } & \multirow{2}{*}{$\begin{array}{c}\mathrm{p} \\
\text { Value }\end{array}$} & \multicolumn{2}{|c|}{ Serious Complication* } & \multirow{2}{*}{$\begin{array}{c}p \\
\text { Value }\end{array}$} \\
\hline & & & & None & Any & & No & Yes & \\
\hline \multicolumn{10}{|c|}{ 30-day complication grade } \\
\hline 0 & 347 (91.8) & 256 (93.1) & \multirow{5}{*}{0.378} & $475(100.0)$ & $128(71.9)$ & \multirow{5}{*}{-} & $549(97.3)$ & $54(60.7)$ & \multirow{5}{*}{-} \\
\hline I & $14(3.7)$ & $4(1.5)$ & & $0(0)$ & $18(10.1)$ & & $15(2.7)$ & $3(3.4)$ & \\
\hline II & $8(2.1)$ & $7(2.5)$ & & $0(0)$ & $15(8.4)$ & & $0(0)$ & $15(16.9)$ & \\
\hline III & $8(2.1)$ & $8(2.9)$ & & $0(0)$ & $16(9.0)$ & & $0(0)$ & $16(18.0)$ & \\
\hline IV & $1(0.3)$ & $0(0)$ & & $0(0)$ & $1(0.6)$ & & $0(0)$ & $1(1.1)$ & \\
\hline Any complication & $102(27.0)$ & $76(27.6)$ & 0.853 & $0(0)$ & $178(100)$ & - & $89(15.8)$ & $89(100.0)$ & - \\
\hline Serious complication & $50(13.2)$ & $39(14.2)$ & 0.726 & $0(0)$ & $89(50.0)$ & - & $0(0)$ & $89(100.0)$ & - \\
\hline \multicolumn{10}{|c|}{ Worst complication grade } \\
\hline 0 & $276(73.0)$ & $199(72.4)$ & \multirow{5}{*}{0.979} & $475(100.0)$ & $0(0)$ & \multirow{5}{*}{-} & 475 (84.2) & $0(0)$ & \multirow{5}{*}{-} \\
\hline 1 & $52(13.8)$ & $37(13.5)$ & & $0(0)$ & $89(50.0)$ & & 89 (15.8) & $0(0)$ & \\
\hline$\|$ & $17(4.5)$ & $13(4.7)$ & & $0(0)$ & 30 (16.9) & & $0(0)$ & $30(33.7)$ & \\
\hline III & $29(7.7)$ & $24(8.7)$ & & $0(0)$ & $53(29.8)$ & & $0(0)$ & $53(59.6)$ & \\
\hline IV & 4 (1.1) & $2(0.7)$ & & $0(0)$ & $6(3.4)$ & & $0(0)$ & $6(6.7)$ & \\
\hline
\end{tabular}

LTAC = long-term acute care facility; NA = not applicable; rehab = rehabilitation; SNF = skilled nursing facility.

All data given as number of patients (\%) unless otherwise indicated. Boldface type indicates statistical significance.

* Serious complications = complications of Grade 2 or higher.

$\dagger$ Senior attending $=20$ or more years post-residency experience.

(OR $1.472,95 \%$ CI $0.883-2.454$ ) nor having surgery after the policy change (OR $1.251,95 \%$ CI $0.748-2.091$ ) was associated with the occurrence of serious complications.

As above, to account for the range of overlap times between overlapping surgeries, we ran a supplemental analysis using the overlapping percentage (overlap time divided by total surgery duration) in our multivariable model of serious complications in place of the overlapping surgery variable. Again, this had no influence on the factors significantly associated with the serious complication rate: overall complications were not significantly associated with overlapping percentage $(\mathrm{p}=0.373)$ and overlapping surgery policy change $(\mathrm{p}=0.471)$.

\section{Wait Time Analysis}

In our multiple linear regression model of surgery wait time (Table 5), craniotomy for aneurysm/vascular malformation $(p=0.037)$, craniotomy for other $(p=0.013)$, transnasal approaches for tumor ( $\mathrm{p}=0.012)$, and miscellaneous cases $(p=0.004)$ were associated with longer surgery wait times compared with our reference procedure of noninstrumented spine. Having surgery performed by attending surgeon number 4 was also associated with a longer wait time compared with our reference attending surgeon number 1 ( $p<0.001)$. Whereas insurance type demonstrated no association with wait time, having an operation after the overlapping surgery policy change was associated with a longer wait time for surgery $(\mathrm{p}<0.001)$.

Because of the nonnormal distribution of wait time data, there were concerns regarding skewing of our results. To account for this, we performed a supplemental analysis on the logarithmic transformation of wait time. In this analysis, procedures occurring after the policy change had significantly longer wait times $(p=0.001)$, with a $\beta$ of 0.147 suggesting an approximately $15 \%$ increased wait time for cases occurring after the implementation of the more restrictive policy.

\section{Discussion}

The recent controversy surrounding the practice of overlapping surgery has spurred a likely long-overdue discussion of the practice among the academic surgical community. Some of the results of this discussion are unarguably beneficial, specifically, greater awareness of the need to educate patients on the practice as a part of informed consent. ${ }^{11}$ Faced with a potential public backlash against overlapping surgery, however, many institutions (including our own) have preemptively implemented sweeping restrictions on the practice. Although previous studies by our group and other neurosurgical centers have suggested that patient safety does not suffer as a result of overlapping surgery, ${ }^{7,16}$ our study represents the first to examine the possible negative repercussions of such policy changes.

In the past year, several studies have suggested that, within the appropriate framework, overlapping surgery may be performed safely without an increase in intraoperative or early postoperative complications. ${ }^{7,9,15,16}$ It is perhaps unsurprising then that our study found no difference in complication rates between overlapping and nonoverlapping cases, either overall or in the subset of serious complications, before or after a more restrictive overlapping surgery policy was implemented.

The primary change in our institution's overlapping surgery policy was the limitation of an attending surgeon's ability to start a second, overlapping case until all critical portions of the first case that could require attending surgeon involvement were completed. Because of the complex 
TABLE 3. Multivariable regression analysis of factors associated with any complication

\begin{tabular}{|c|c|c|}
\hline Variable & OR $(95 \% \mathrm{Cl})$ & p Value \\
\hline Surgical category & & 0.041 \\
\hline Simple craniotomy for tumor & 1.00 (ref) & NA \\
\hline Complex craniotomy for tumor & $1.584(0.637-3.939)$ & 0.323 \\
\hline $\begin{array}{l}\text { Craniotomy for aneurysm/ } \\
\text { vascular malformation }\end{array}$ & $1.187(0.440-3.205)$ & 0.735 \\
\hline Craniotomy for other & $0.518(0.191-1.401)$ & 0.195 \\
\hline Burr hole craniotomy & $1.819(0.562-5.890)$ & 0.318 \\
\hline Noninstrumented spine & $0.378(0.139-1.025)$ & 0.056 \\
\hline Instrumented spine & $0.824(0.446-1.521)$ & 0.536 \\
\hline Transnasal approach for tumor & $2.088(0.943-4.622)$ & 0.069 \\
\hline Shunt & $0.755(0.323-1.769)$ & 0.518 \\
\hline Miscellaneous & $1.008(0.456-2.229)$ & 0.984 \\
\hline Overlapping surgery & $1.072(0.710-1.620)$ & 0.741 \\
\hline Post-policy change & $1.057(0.700-1.596)$ & 0.792 \\
\hline Surgery length (mins) & $1.003(1.000-1.005)$ & 0.023 \\
\hline Hepatobiliary comorbidity & $2.628(1.032-6.693)$ & 0.043 \\
\hline ASA Physical Status Class & & $<0.001$ \\
\hline | or II & 1.00 (ref) & NA \\
\hline III & $1.659(1.081-2.547)$ & 0.021 \\
\hline IV or V & $7.780(3.754-16.124)$ & $<0.001$ \\
\hline Estimated blood loss (ml) & $1.002(1.001-1.003)$ & 0.001 \\
\hline
\end{tabular}

Ref = reference.

Boldface type indicates statistical significance.

and unpredictable nature of many neurosurgical procedures, this meant that a large proportion of operations could no longer be performed in an overlapping fashion at all. This also precluded any shorter, minor procedures from being started and completed while a more involved case was being set up. Although this policy shift was effective in curtailing overlapping surgery at our institution, with a reduction in overlapping surgery rates of nearly $42 \%$, our study suggests that this change was not without consequences. The absolute number of cases for the 4 attending surgeons studied during each 5-month span fell by more than $27 \%$ after the revised policy implementation. In terms of resident education, the number of cases in which a postresidency fellow acted as an assistant increased significantly after the increased restrictions were implemented, from slightly less than $12 \%$ of cases to over a third. The increase in fellow involvement necessarily limits the ability of resident trainees to act as the lead assistant provider in such cases.

Finally, with regard to patient wait times for neurosurgical procedures, our study suggests that the implementation of the more restrictive overlapping surgery policy was associated with a significant increase in delay from the decision to perform surgery to the operation itself. Based on our multiple linear regression model, even controlling for surgery type, individual attending surgeon, and insurance type, patients undergoing surgery after the policy change waited more than 11 days longer than patients waited prior to the change.

Our study has several limitations. The retrospective na-
TABLE 4. Multivariable regression analysis of factors associated with serious complications

\begin{tabular}{lcc}
\hline \multicolumn{1}{c}{ Variable } & OR $(95 \%$ Cl $)$ & p Value \\
\hline Surgery category & & 0.049 \\
\hline Simple craniotomy for tumor & $1.00($ ref $)$ & NA \\
\hline Complex craniotomy for tumor & $0.852(0.318-2.279)$ & 0.749 \\
\hline $\begin{array}{c}\text { Craniotomy for aneurysm/ } \\
\text { vascular malformation }\end{array}$ & $0.689(0.233-2.031)$ & 0.499 \\
\hline Craniotomy for other & $0.533(0.176-1.612)$ & 0.265 \\
\hline Burr hole craniotomy & $2.229(0.653-7.610)$ & 0.201 \\
\hline Noninstrumented spine & $0.257(0.070-0.946)$ & 0.041 \\
\hline Instrumented spine & $0.279(0.122-0.638)$ & $\mathbf{0 . 0 0 2}$ \\
\hline Transnasal approach for tumor & $0.644(0.215-1.928)$ & 0.431 \\
\hline Shunt & $0.716(0.272-1.886)$ & 0.499 \\
\hline Miscellaneous & $0.869(0.351-2.148)$ & 0.761 \\
\hline Overlapping surgery & $1.472(0.883-2.454)$ & 0.138 \\
\hline Post-policy change & $1.251(0.748-2.091)$ & 0.393 \\
\hline Surgery length (mins) & $1.004(1.002-1.006)$ & 0.001 \\
\hline ASA Physical Status Class & & $<0.001$ \\
\hline I or II & $1.00($ ref $)$ & NA \\
\hline III & $1.702(0.973-2.975)$ & 0.062 \\
\hline IV or V & $5.995(2.759-13.024)$ & $<0.001$ \\
\hline Boldace tye indcates stastical & &
\end{tabular}

Boldface type indicates statistical significance.

ture of the analysis makes it vulnerable to confounding. Although we attempted to control for as many demographic, procedure-specific, and attending surgeon-specific variables as possible, it is inevitable that some important factors were missed. Because of the controversial nature of overlapping surgery, it would likely be exceedingly difficult at this point to complete our study in a prospective or randomized manner. Although the policy change did appear to be associated with increased surgery wait times, it is unknown whether this delay had any influence on outcomes. Our definition of overlapping surgery was broad, based on any period of overlap from the start of anesthesia to the end of anesthesia. This raises the possibility that some cases that we deemed "overlapping" may only have overlapped briefly, or only during induction/conclusion of anesthesia, possibly skewing our results. As described earlier, the nonnormal distribution of our wait time variable makes it possible that a multivariable linear regression model could overestimate the influence of our independent variables, although the sustained association of the policy change with significantly increased wait time in our logarithmic transformation model is reassuring. Finally, our study is limited to a single center and our sample size is limited. This reduces the generalizability of our findings to other practices and increases our study's vulnerability to sampling error, a possible explanation for the demographic changes noted in our pre- and post-policy change groups.

\section{Conclusions}

Policies aimed at limiting overlapping surgery may be effectively and rapidly implemented in neurosurgical practices in an effort to stem public concerns about potentially 
TABLE 5. Multiple linear regression analysis of surgical wait times

\begin{tabular}{lccc}
\hline \multicolumn{1}{c}{ Variable } & B (SE) in Days & $\beta$ & p Value \\
\hline Surgery category & & & \\
\hline Noninstrumented spine & Ref & Ref & NA \\
\hline Simple craniotomy for tumor & $14.036(9.074)$ & 0.147 & 0.123 \\
\hline Complex craniotomy for tumor & $12.337(10.197)$ & 0.086 & 0.227 \\
\hline $\begin{array}{l}\text { Craniotomy for aneurysm/ } \\
\text { vascular malformation }\end{array}$ & $22.887(10.965)$ & 0.120 & 0.037 \\
\hline Craniotomy for other & $24.059(9.620)$ & 0.164 & 0.013 \\
\hline Burr hole craniotomy & $5.186(17.962)$ & 0.014 & 0.773 \\
\hline Instrumented spine & $5.796(4.874)$ & 0.071 & 0.235 \\
\hline Transnasal approach for tumor & $26.533(10.465)$ & 0.223 & 0.012 \\
\hline Shunt & $6.485(8.762)$ & 0.053 & 0.460 \\
\hline Miscellaneous & $23.072(8.015)$ & 0.207 & 0.004 \\
\hline Attending no. & & & \\
\hline 1 & Ref & Ref & NA \\
\hline 2 & $-9.089(6.017)$ & -0.090 & 0.132 \\
\hline 3 & $-0.203(6.275)$ & -0.002 & 0.974 \\
\hline 4 & $32.903(7.258)$ & 0.460 & $<0.001$ \\
\hline Insurance type & & & \\
\hline Medicare & Ref & Ref & NA \\
\hline Private & $-3.094(3.279)$ & -0.044 & 0.346 \\
\hline Medicaid/self-pay & $4.069(5.696)$ & 0.033 & 0.475 \\
\hline Post-policy change & $11.149(3.082)$ & 0.159 & $<0.001$ \\
\hline SE standard rivar & & &
\end{tabular}

$\mathrm{SE}=$ standard error.

Boldface type indicates statistical significance.

negative consequences. The data in this study show that policy changes regarding overlapping surgery will have significant consequences with respect to resident surgeon education, surgeon availability, and wait times for patient access to elective surgery. At our institution, these policy changes did not result in any positive impact on complication rates or patient outcomes. Further investigations at other centers are needed to better define the overall impact of overlapping surgery policy changes on training, access to care, and patient outcomes.

\section{References}

1. Abelson J, Saltzman J, Kowalczyk L: Concurrent surgeries come under new scrutiny. Boston Globe. December 20, 2015. (http://www.bostonglobe.com/metro/2015/12/19/concernover-double-booked-surgeries-emerges-national-issue-forhospitals/6IjRw2WkDYdt5oZljpajcO/story.html) [Accessed on June 21, 2017]

2. Abelson J, Saltzman J, Kowalczyk L, Allen S: Clash in the name of care. Boston Globe. (https://apps.bostonglobe.com/ spotlight/clash-in-the-name-of-care/story.) [Accessed on June 21, 2017]

3. American Association of Neurological Surgeons: Position Statement on Intraoperative Responsibility of the Primary Neurosurgeon. (http://www.aans.org/ /link.aspx?_id= 8FA6415D87CE49D49052F0474FD39CFF\&_z=z) [Accessed June 21, 2017]

4. American College of Surgeons: Statement on Principles. (https://www.facs.org/about-acs/statements/ stonprin\#anchor172771) [Accessed June 21, 2017]
5. Beasley GM, Pappas TN, Kirk AD: Procedure delegation by attending surgeons performing concurrent operations in academic medical centers: balancing safety and efficiency. Ann Surg 261:1044-1045, 2015

6. Cole E, Hopman W, Kawakami J: High resolution analysis of wait times and factors affecting surgical expediency. Can Urol Assoc J 5:13-17, 2011

7. Guan J, Brock AA, Karsy M, Couldwell WT, Schmidt $\mathrm{MH}$, Kestle JR, et al: Managing overlapping surgery: an analysis of 1018 neurosurgical and spine cases. J Neurosurg [epub ahead of print December 2, 2016. DOI: 10.3171/2016.8.JNS161226]

8. Guan J, Karsy M, Brock A, Couldwell W, Schmidt R: Overlapping surgery: a review of the controversy, the evidence, and future directions. Clin Neurosurg 64(CN Suppl 1):110113,2017

9. Hyder JA, Hanson KT, Storlie CB, Glasgow A, Madde NR, Brown MJ, et al: Safety of overlapping surgery at a highvolume referral center. Ann Surg 265:639-644, 2017

10. Landriel Ibañez FA, Hem S, Ajler P, Vecchi E, Ciraolo C, Baccanelli M, et al: A new classification of complications in neurosurgery. World Neurosurg 75:709-715, 604-611, 2011

11. Langerman A: Concurrent surgery and informed consent. JAMA Surg 151:601-602, 2016

12. Mello MM, Livingston EH: Managing the risks of concurrent surgeries. JAMA 315:1563-1564, 2016

13. United States Senate Finance Committee: Concurrent and Overlapping Surgeries: Additional Measures Warranted. (https://www.finance.senate.gov/imo/media/doc/ Concurrent $\% 20$ Surgeries\%20Report \%20Final.pdf) [Accessed June 21, 2017]

14. Yount KW, Gillen JR, Kron IL, Kern JA, Kozower BD, Ailawadi G, et al: Attendings' performing simultaneous operations in academic cardiothoracic surgery doe not increase operative duration or negatively affect patient outcomes, presented at the 96th American Association for Thoracic Surgery Annual Meeting, May 14-18, 2016, Baltimore. (http://t.aats.org/annualmeeting/Program-Books/2014/2.cgi) [Accessed June 21, 2017]

15. Zhang AL, Sing DC, Dang DY, Ma CB, Black D, Vail TP, et al: Overlapping surgery in the ambulatory orthopaedic setting. J Bone Joint Surg Am 98:1859-1867, 2016

16. Zygourakis CC, Lee J, Barba J, Lobo E, Lawton MT: Performing concurrent operations in academic vascular neurosurgery does not affect patient outcomes. J Neurosurg [epub ahead of print January 20, 2017. DOI: 10.3171/2016.6.JNS15822]

\section{Disclosures}

The authors report the following. Dr. Dailey is a consultant for Medtronic, has received royalties and research support from Biomet, and has received speaking fees from AONA. Dr. Jensen is a consultant for Medtronic and Pharmaco-Kinesis, and is on advisory boards for Pharmoco-Kinesis and Varian.

\section{Author Contributions}

Conception and design: Schmidt, Guan, Karsy, Brock. Analysis and interpretation of data: Guan. Drafting the article: Schmidt, Guan, Karsy, Brock. Critically revising the article: Schmidt, Couldwell, Kestle, Jensen, Dailey. Reviewed submitted version of manuscript: Schmidt, Guan. Approved the final version of the manuscript on behalf of all authors: Schmidt.

\section{Correspondence}

Richard H. Schmidt, Department of Neurosurgery, Clinical Neurosciences Center, University of Utah, 175 N Medical Dr. East, Salt Lake City, UT 84132. email: neuropub@hsc.utah.edu. 